\title{
Maladie de Rendu-Osler révélée par des télangiectasies oro-faciales : à propos d'un cas
}

\section{Curien R, Guillet J, Aldosa j, Maschino F. (Université de Lorraine, Faculté d'Odontologie, Nancy)}

La maladie de Rendu Osler, ou Télangiectasie Hémorragique Héréditaire, est une maladie vasculaire génétique de transmission autosomique dominante, et caractérisée par des angiodysplasies de sièges multiples. Son potentiel de gravité réside dans l'apparition possible de malformations artério-veineuses viscérales (Te Vedldhuis et al, 2008 ; Plauchu, Dupuis-Girod, 2009).

Une patiente de 56 ans consulte le service d'odontologie en raison de douleurs au niveau d'une 18 cariée. Au décours de l'examen sont constatées de multiples télangiectasies disparaissant à la vitropression et constellant la muqueuse orale et le pourtour labial. D'après la patiente ces lésions sont présentes depuis de nombreuses années. D'autres lésions similaires sont retrouvées au niveau du cou, des poignets et de la pulpe des doigts. La patiente évoque par ailleurs de fréquents épistaxis et gingivorragies ainsi qu'une notion de terrain familial (plusieurs membres de sa famille sujets aux épistaxis et télangiectasies présentes chez sa soeur). Un bilan biologique complémentaire (NFS, bilan d'hémostase, bilan hépatique et bilan martial) est prescrit et ne démontre aucune anomalie

La présence de deux critères diagnostics (télangiectasies de la peau et des muqueuses orales, épistaxis spontanés et récurrents), ainsi que la suspicion d'une hérédité familiale, évoque fortement une maladie de Rendu Osler.

La patiente est donc adressée pour bilan et prise en charge auprès du Centre de Compétence pour la Maladie de Rendu-Osler. Le bilan pratiqué confirme le diagnostic de Maladie de Rendu-Osler, et met en évidence deux malformations artério-veineuses à l'étage thoracique relevant pour le moment d'une simple surveillance.

Des télangiectasies orales et faciales sont fréquemment retrouvées dans le cadre de la maladie de Rendu-0sler (Te Vedldhuis et al, 2008 ; Da silva et al, 2009). La présence de télangiectasies multiples dans la sphère orofaciale doit toujours faire évoquer la maladie de Rendu-0sler, et doit inciter à faire pratiquer un bilan pulmonaire, neurologique, digestif et hépatique afin de dépister des complications potentiellement graves.

CURIEN Rémi

remi.curien@yahoo.fr 